\title{
Growth factors in Anorexia Nervosa: protocol for a systematic review and meta-analysis of cross-sectional and longitudinal data
}

\author{
Johanna Keeler $^{1 *}$, Janet Treasure ${ }^{1}$, Rosemarie Keeler ${ }^{2}$ and Hubertus Himmerich ${ }^{1}$, \\ Author affiliations \\ ${ }^{1}$ Department of Psychological Medicine, Institute of Psychiatry, Psychology \& Neuroscience, King's \\ College London, London, UK \\ ${ }^{2}$ The Open University, Walton Hall, Kents Hill, Milton Keynes, UK
}

\section{Corresponding author*}

Johanna Keeler

Department of Psychological Medicine, Institute of Psychiatry, Psychology \& Neuroscience, King's College London, London, UK

Email: johanna.keeler@kcl.ac.uk

\section{Keywords}

anorexia nervosa, growth factor, brain derived neurotrophic factor, systematic review, meta-analysis

\section{Registration details}

This protocol was registered with the Open Science Framework on 4 February 2021.

\begin{abstract}
Introduction

Anorexia Nervosa (AN) is a serious mental health condition, which commonly follows a chronic course. Recent evidence suggests SE-AN may be underpinned by an interaction between genetic risk factors, the endocrinological, immune and metabolic systems and deficiencies in neural structures. Previous meta-analyses have identified increases in certain pro-inflammatory cytokines in AN, which may contribute to neuroinflammation. However, there has not yet been an investigation of growth factor concentrations in individuals with AN. We aim to conduct a systematic review and metaanalysis of growth factor concentrations in individuals with AN compared to healthy individuals, and of individuals longitudinally (i.e. after an improvement in weight and/or symptoms).

\section{Methods and analysis}

We will use a systematic review and meta-analysis based on the Preferred Reporting Items for Systematic Reviews and Meta-Analyses (PRISMA) guidelines (Moher et al., 2009). Studies of any design in populations of AN with outcomes pertaining to serum, plasma or cerebrospinal growth factor concentrations will be located using an electronic database search of PudMed, ISI Web of Science Core Collection and MEDLINE. Two reviewers will independently screen titles, abstracts and full-texts and chart data of eligible studies. Study characteristics will be summarised during data analysis. Reported outcomes will include standardised mean differences between groups (AN vs. healthy controls, AN longitudinally) of individual growth factors.
\end{abstract}




\section{INTRODUCTION}

Anorexia nervosa (AN) is a serious and persistent psychiatric disorder, characterised by disturbed body image and an intense fear of weight gain, leading to severe dietary restriction ${ }_{2}$ other weight loss behaviours, and significantly low weight (American Psychiatric Association, 2013). The disorder is associated with high relapse rates, with approximately $30 \%$ of individuals having an illness duration of greater than 15 years (Støving, Andries, Brixen, Bilenberg \& Hørder, 2011). Moreover, only 31\% reach recovery after 9 years (Eddy et al., 2017). A major contributor to the high rates of relapse and chronicity of the disorder is that the factors contributing to the chronic phase of the illness are not yet clear. However, there have been recent advances in our understanding, with genetic studies indicating a complex link between genes, environmental and nutritional factors (Watson et al., 2019; Hubel, 2019; Himmerich et al., 2019). In particular, the chronic form of AN is underpinned by the interaction between genetic risk factors and secondary physiological alterations involving the immune, endocrine and metabolic systems and neural systems (Duriez et al., 2019; Himmerich et al., 2019).

Neuroendocrine changes are observed over the course of AN, many of which are a function of the body's adaptation to low weight. For example, lower concentrations of insulin-like growth factor-1 (IGF-1), hypercortisolaemia, decreases in leptin, amylin, sinsulin and incretins and increases in ghrelin, peptide YY and adiponectin have been observed (Misra \& Klibanski, 2015). These endocrine changes can result in alterations in bone metabolism (e.g. low bone mass), body composition, reproductive function, psychopathology and neurocognition in AN (Misra \& Klibanski, 2010; Misra \& Klibanski, 2015). Moreover, AN is associated with neuroendocrine changes associated with neuroinflammation, such as increased pro-inflammatory cytokine production (Dalton et al., 2018) and reductions in key growth factors important in neuroplasticity such as brain-derived neurotrophic factor (BDNF; Brandys, Kas, Van Elburg, Campbell \& Adan, 2010).

Neuroinflammation has been implicated in the development and maintenance of several brain and psychiatric diseases (Lyman, Lloyd, Ji, Vizcaychipi \& Ma, 2014). Prolonged neuroinflammation contributes to structural and functional brain changes along the course of the illness progression; otherwise known as neuroprogression (Halaris, 2018). It is thought to be mediated by increases in certain pro-inflammatory cytokines (e.g. TNF-a, interleukin [IL]-6, IL1- $\beta$ ) together with reductions in the production of certain growth factors (e.g. BDNF, vascular endothelial growth factor [VEGF], glial cell line-derived neurotrophic factor [GDNF]; see Levy et al., 2018 for a review). Growth factors such as BDNF, IGF-1 and VEGF are important for the regulation of adult hippocampal neurogenesis (Rossi, Angelucci \& Constantin, 2006; Fabel, Fabel \& Tam, 2003); a key component of neuroplasticity. Pro-inflammatory cytokines such as IL1- $\beta$ reduce BDNF expression in neural regions in rodents, specifically in the hippocampus (Lapchak et al., 1993). Moreover, IGF-1 is thought to provide neuroprotection, and decreases due to pro-inflammatory cytokines such as TNF-a may contribute to neuroinflammation (Labandeira-Garcia et al., 2017).

Neuroprogression is a concept that has recently been introduced in the context of AN, due to many of the aforementioned neuroendocrine changes observed in AN that are indicative of neuroinflammation (see the Cognitive Interpersonal Model, Treasure et al., 2020; Treasure \& Schmidt, 2013). For example, meta-analytic findings showed decreases in BDNF in AN with a large effect size (Brandys et al., 2010). Past research from our group has indicated reductions in BDNF, VEGF-A and tumor necrosis factor- $\beta$ in individuals with AN (Dalton et al., 2018). These are coupled with increases in certain pro-inflammatory cytokines, such as IL-6, IL-15 (Dalton et al., 2018), IL1- $\beta$ and TNF-a (Solmi et al., 2015). Moreover, serum IGF-1 has been found to be decreased in AN, which is partially reversed after refeeding (Counts, Gwirtsman, Carlsson, Lesem \& Cutler, 1992).

Recent meta-analyses have explored cytokine levels in AN and other EDs (Dalton et al., 2018; Solmi et al., 2015), together with more explorations of specific growth factors such as BDNF (Brandys et al., 2010), However, concentrations of many other growth factors in AN have not yet been systematically collated in this patient group. Therefore, the primary aim of this meta-analysis will be 
to synthesise studies investigating growth factor concentrations in people with $\mathrm{AN}$, both in comparison to healthy controls and longitudinally.

\section{Objectives}

i) Examine whether growth factor concentrations differ between individuals with $\mathrm{AN}$ and healthy individuals

ii) Examine whether growth factor concentrations change longitudinally in $\mathrm{AN}$, as a function of weight gain or symptom improvement

\section{METHODS AND ANALYSIS}

\section{Study design}

This systematic review and meta-analysis will be conducted according to the Preferred Reporting Items for Systematic Reviews and Meta-Analyses (PRISMA) guidelines (Moher et al., 2009).

\section{Eligibility criteria}

Studies in any language of any study design that assess growth factor concentrations in the serum, plasma or cerebrospinal fluid of individuals with a current or past Diagnostic and Statistical Manual of Mental Disorders (American Psychiatric Association, 2013), or International Statistical Classification of Diseases (ICD; World Health Organisation, 2018) diagnosis of AN/a-typical AN will be eligible. Publications will be included if they report cross-sectional comparisons of growth factor concentrations between those with AN and a comparator group or longitudinal assessments of individuals with AN. Longitudinal studies will be included if growth factor concentrations are measured at a minimum of two time-points and if eating disorder symptomatology and BMI are also assessed. Studies using participants with co-morbid psychiatric diagnoses will be included. There will be no BMI cut-off for inclusion in this study, in order to include populations with AN at varying clinical severities and at various stages of illness. Studies using animal samples will be excluded.

The exclusion criteria are as follows: i) studies using animal samples; ii) participants have an organic cause for their eating disorder e.g. cancer, immunological conditions, genetic disorder, etc.; iii) the study does not report group comparisons or longitudinal measurements of growth factors; iv) review articles, meta-analyses, conference proceedings/abstracts, unpublished theses and book chapters.

\section{Information sources and search strategy}

Two reviewers (J.K. and R.K.) will independently search databases. The literature will be searched using three electronic databases (PudMed, ISI Web of Science Core Collection and MEDLINE), which will be supplemented by additional hand searches through reference lists. Journals will be searched from time of inception. The following search terms will be used:

(((growth factor) OR (neurotrophic factor) OR (stimulating factor) OR (differentiation factor)) AND ((anorexia) OR (anorexia nervosa))) NOT ((cancer) OR (review) OR (letter) OR (mice) OR (rats)).

Searches will be limited to the titles/abstracts and to studies performed on human subjects. We will manually scan titles and abstracts for related terms (e.g. endocrinology).

The review will commence in February 2021 and will continue until August 2021. The final paper will follow the Preferred Reporting Items for Systematic Reviews and Meta-Analyses (Moher et al., 2009). 


\section{Source selection}

Identified papers will be imported into a reference manager (e.g. EndNote) where duplicates will be removed. Further to this, titles and abstracts will be independently screened by two reviewers. The full article will be retrieved for further assessment if the information in the title or abstract suggests that the study assessed serum, plasma or CSF growth factor levels in individuals with AN either (1) longitudinally or (2) in comparison to a healthy control group. Studies that are not available on the Internet or through libraries will be obtained by contacting the study authors.

Following this, identified studies will be examined in more detail. Reasons for exclusions will be recorded in a spreadsheet. In the case of any discrepancies in study selection, discussion with two experienced authors (H.H. and J.T.) will resolve any conflicts.

\section{Data charting}

Data from each study will be extracted independently by the main investigator (J.K.) and checked by another reviewer (R.K.), using a data extraction spreadsheet. Data will only be charted for participants with AN at multiple timepoints, or those with $\mathrm{AN}$ and healthy controls.

The following data will be charted: publication identifiers (e.g. journal, year, first author); country of origin; design; study objective; methodology; sample characteristics (e.g. mean age, ethnic background, diagnosis, diagnostic tool, sample size); relevant findings. We will chart the following data for outcomes: parameters of interest, measurement methods and concentrations of growth factors. Data that are not available on the published papers will be obtained by contacting the study authors.

\section{Synthesis of results}

All meta-analyses will be conducted in Stata (StataCorp, 2017). Individual meta-analyses will be performed for each identified growth factor (with 2 or more available studies). For studies that included more than one diagnostic group (e.g. AN and a-typical AN participants) or AN subtype, the means and standard deviations will be pooled. Reported standard error means will be converted to SDs. The standardised mean difference will be used as the summary statistic, which is a metric for expressing the size of the effect in each study (e.g. AN vs HC), relative to the variability in the study. A random-effects model will be used for all meta-analyses, using the DerSimonian \& Laird method (DerSimonian and Laird, 1986). SMDs will be pooled using the Hedges method (Hedges, 1981). We will explore the possibility of subgroup analyses based on AN subtype (restrictive [AN-R] vs bingepurge $[\mathrm{AN}-\mathrm{BP}])$.

Heterogeneity between studies will be assessed by calculating Higgins $\mathrm{I}^{2}$ (Higgins et al., 2003), which measures the percentage of total variation in standardised mean difference across studies due to heterogeneity. Between-study heterogeneity will be considered as high if $\mathrm{I}^{2}$ is above $50 \%$.

Publication bias will be assessed using the Duval and Tweedie trim and fill method (Duval and Tweedie, 2000). This method identifies smaller studies causing funnel plot asymmetry and adjusts for this asymmetry. SMDs will be re-estimated after adjusting for missing studies. The Egger's test for small study effects will also be used.

\section{Risk of bias assessment}

We will use the Newcastle-Ottawa Scale (Wells et al., 2000) to assess the quality of the observational studies, recommended by the Cochrane collaboration (Cochrane Handbook for Systematic Review of Interventions, 2012).

\section{AUTHOR CONTRIBUTIONS}

JK drafted the protocol. All authors reviewed and provided feedback on the study design and manuscript. All authors read and approved the protocol before publishing. 


\section{FUNDING STATEMENT}

Johanna Keeler is receiving funding through a Medical Research Council stipend for the Doctoral Training Programme. Hubertus Himmerich has received salary support from the NIHR BRC at the South London and Maudsley NHS Foundation Trust (SLaM) and KCL.

\section{REFERENCES}

American Psychiatric Association. (2013). Feeding and Eating Disorders. In Diagnostic and statistical manual of mental disorders (5th ed.). https://dsm.psychiatryonline.org/doi/full/10.1176/appi.books.9780890425596.dsm10

Brandys, M. K., Kas, M. J., van Elburg, A. A., Campbell, I. C., \& Adan, R. A. (2011). A metaanalysis of circulating BDNF concentrations in anorexia nervosa. The World Journal of Biological Psychiatry, 12(6), 444-454.

Counts, D. R., Gwirtsman, H. A. R. R. Y., Carlsson, L. M., Lesem, M., \& Cutler Jr, G. B. (1992). The effect of anorexia nervosa and refeeding on growth hormone-binding protein, the insulin-like growth factors (IGFs), and the IGF-binding proteins. The Journal of Clinical Endocrinology \& Metabolism, 75(3), 762-767.

Dalton, B., Bartholdy, S., Robinson, L., Solmi, M., Ibrahim, M. A., Breen, G., ... \& Himmerich, H. (2018). A meta-analysis of cytokine concentrations in eating disorders. Journal of psychiatric research, 103, 252-264.

Duriez, P., Ramoz, N., Gorwood, P., Viltart, O., \& Tolle, V. (2019). A metabolic perspective on reward abnormalities in anorexia nervosa. Trends in Endocrinology \& Metabolism, 30(12), 915-928.

Eddy, K. T., Tabri, N., Thomas, J. J., Murray, H. B., Keshaviah, A., Hastings, E., ... \& Keel, P. K. (2017). Recovery from anorexia nervosa and bulimia nervosa at 22-year follow-up. The Journal of clinical psychiatry, 78(2), 184-189.

Fabel, K., Fabel, K., Tam, B., Kaufer, D., Baiker, A., Simmons, N., ... \& Palmer, T. D. (2003). VEGF is necessary for exercise-induced adult hippocampal neurogenesis. European Journal of Neuroscience, 18(10), 2803-2812.

Halaris, A. (2018). Neuroinflammation and neurotoxicity contribute to neuroprogression in neurological and psychiatric disorders. Future Neurology, 13(2), 59-69.

Himmerich, H., Hotopf, M., Shetty, H., Schmidt, U., Treasure, J., Hayes, R. D., ... \& Chang, C. K. (2019). Psychiatric comorbidity as a risk factor for mortality in people with anorexia nervosa. European archives of psychiatry and clinical neuroscience, 269(3), 351-359.

Hübel, C., Yilmaz, Z., Schaumberg, K. E., Breithaupt, L., Hunjan, A., Horne, E., ... \& Breen, G. (2019). Body composition in anorexia nervosa: Meta-analysis and meta-regression of crosssectional and longitudinal studies. International Journal of Eating Disorders, 52(11), 12051223.

Labandeira-Garcia, J. L., Costa-Besada, M. A., Labandeira, C. M., Villar-Cheda, B., \& RodríguezPerez, A. I. (2017). Insulin-like growth factor-1 and neuroinflammation. Frontiers in aging neuroscience, 9, 365 .

Lapchak, P. A., Araujo, D. M., \& Hefti, F. (1993). Systemic interleukin-1 beta decreases brainderived neurotrophic factor messenger RNA expression in the rat hippocampal formation. Neuroscience, 53(2), 297-301.

Levy, M. J., Boulle, F., Steinbusch, H. W., van den Hove, D. L., Kenis, G., \& Lanfumey, L. (2018). Neurotrophic factors and neuroplasticity pathways in the pathophysiology and treatment of depression. Psychopharmacology, 235(8), 2195-2220. 
Lyman, M., Lloyd, D. G., Ji, X., Vizcaychipi, M. P., \& Ma, D. (2014). Neuroinflammation: the role and consequences. Neuroscience research, 79, 1-12.

Misra, M., \& Klibanski, A. (2010). Neuroendocrine consequences of anorexia nervosa in adolescents. Pediatric Neuroendocrinology, 17, 197-214.

Misra, M., \& Klibanski, A. (2015). Eating Disorders and Their Effects on Bone Health. In Nutrition and Bone Health (pp. 599-616). Humana Press, New York, NY.

Rossi, C., Angelucci, A., Costantin, L., Braschi, C., Mazzantini, M., Babbini, F., ... \& Caleo, M. (2006). Brain-derived neurotrophic factor (BDNF) is required for the enhancement of hippocampal neurogenesis following environmental enrichment. European Journal of Neuroscience, 24(7), 1850-1856.

Solmi, M., Veronese, N., Favaro, A., Santonastaso, P., Manzato, E., Sergi, G., \& Correll, C. U. (2015). Inflammatory cytokines and anorexia nervosa: A meta-analysis of cross-sectional and longitudinal studies. Psychoneuroendocrinology, 51, 237-252.

Støving, R. K., Andries, A., Brixen, K., Bilenberg, N., \& Hørder, K. (2011). Gender differences in outcome of eating disorders: a retrospective cohort study. Psychiatry research, 186(2-3), 362366.

Treasure, J., \& Schmidt, U. (2013). The cognitive-interpersonal maintenance model of anorexia nervosa revisited: a summary of the evidence for cognitive, socio-emotional and interpersonal predisposing and perpetuating factors. Journal of eating disorders, 1(1), 1-10.

Treasure, J., Willmott, D., Ambwani, S., Cardi, V., Clark Bryan, D., Rowlands, K., \& Schmidt, U. (2020). Cognitive interpersonal model for anorexia nervosa revisited: The perpetuating factors that contribute to the development of the severe and enduring illness. Journal of clinical medicine, 9(3), 630.

Watson, H. J., Yilmaz, Z., Thornton, L. M., Hübel, C., Coleman, J. R., Gaspar, H. A., ... \& Slagboom, P. E. (2019). Genome-wide association study identifies eight risk loci and implicates metabopsychiatric origins for anorexia nervosa. Nature genetics, 51(8), 1207-1214.

Wells, G. A., Shea, B., O'Connell, D. A., Peterson, J., Welch, V., Losos, M., \& Tugwell, P. (2000). The Newcastle-Ottawa Scale (NOS) for assessing the quality of nonrandomised studies in metaanalyses.

World Health Organization. (2018). International classification of diseases for mortality and morbidity statistics (11th Revision). Retrieved from https://icd.who.int/browse11/1-m/en 